\title{
Multiple solutions of discrete Schrödinger equations with growing potentials
}

\author{
Liqian Jia and Guanwei Chen*
}

${ }^{*}$ Correspondence: guanweic@163.com

School of Mathematical Sciences, University of Jinan, Jinan, Shandong Province 250022, P.R. China

\begin{abstract}
Under some weaker conditions than elsewhere, we obtain infinitely many homoclinic solutions for a class of discrete Schrödinger equations in infinite $m$ dimensional lattices with nonlinearities being superlinear at infinity by using variational methods. Our result extends some existing results in the literature.
\end{abstract}

MSC: 35Q51; 35Q55; 39A12; 39A70

Keywords: discrete nonlinear Schrödinger equations; variational methods; superlinear; homoclinic solutions

\section{Introduction and main results}

The discrete nonlinear Schrödinger equation is one of the most important discrete models, which plays an important role in many fields; for example, in biomolecular chains [1], nonlinear optics [2], Bose-Einstein condensates [3], and so on. In recent decades, a lot of results have been achieved in the study of homoclinic solutions for periodic discrete nonlinear Schrödinger equations; see [4-14], and so on. But we notice that there are only a few results of non-periodic discrete nonlinear Schrödinger equations, such as [15-23]. The authors of $[16,17,19,22]$ studied the case in infinite one dimensional lattices (i.e., $n \in Z$ ), but the authors of $[15,18,20,21,23]$ studied the case in infinite $m$ dimensional lattices (i.e., $n \in Z^{m}$ ).

Inspired by the above results, we will study homoclinic solutions of the following nonperiodic discrete nonlinear equation in infinite $m$ dimensional lattices by more general conditions than some existing results:

$$
-\Delta u_{n}+v_{n} u_{n}-\omega u_{n}=f_{n}\left(u_{n}\right), \quad n \in Z^{m},
$$

where

$$
\begin{aligned}
\Delta u_{n}= & u_{\left(n_{1}+1, n_{2}, \ldots, n_{m}\right)}+u_{\left(n_{1}, n_{2}+1, \ldots, n_{m}\right)}+\cdots+u_{\left(n_{1}, n_{2}, \ldots, n_{m}+1\right)}-2 m u_{\left(n_{1}, n_{2}, \ldots, n_{m}\right)} \\
& +u_{\left(n_{1}-1, n_{2}, \ldots, n_{m}\right)}+u_{\left(n_{1}, n_{2}-1, \ldots, n_{m}\right)}+u_{\left(n_{1}, n_{2}, \ldots, n_{m}-1\right)}
\end{aligned}
$$

is the discrete Laplace operator in $m$ dimensional space, $\omega \in R, V=\left\{v_{n}\right\}_{n \in Z^{m}}$, and $\left\{u_{n}\right\}_{n \in Z^{m}}$ are sequences of real numbers, and the nonlinearities $f_{n}$ satisfy the condition:

$$
f_{n}\left(e^{i \omega} s\right)=e^{i \omega} f_{n}(s), \quad \forall \omega \in R, \forall(n, s) \in Z^{m} \times R .
$$

(c) 2016 Jia and Chen. This article is distributed under the terms of the Creative Commons Attribution 4.0 International License (http://creativecommons.org/licenses/by/4.0/), which permits unrestricted use, distribution, and reproduction in any medium, provided you give appropriate credit to the original author(s) and the source, provide a link to the Creative Commons license, and indicate if changes were made. 
As usual, homoclinic solutions of equation (1.1) satisfy the following boundary condition:

$$
\lim _{|n|=\left|n_{1}\right|+\left|n_{2}\right|+\cdots+\left|n_{m}\right| \rightarrow \infty} u_{n}=0
$$

Here we are interested in the existence of infinitely many nontrivial homoclinic solutions for (1.1) (' $u$ is nontrivial' means $u_{n} \not \equiv 0$ ). The problem (1.1) comes from the study of standing waves for the discrete nonlinear Schrödinger equation

$$
i \dot{\psi}_{n}=-\Delta \psi_{n}+v_{n} \psi_{n}-f_{n}\left(\psi_{n}\right), \quad n \in Z^{m}
$$

By the definition of standing waves $\left(\psi_{n}=u_{n} e^{-i \omega t}\right.$ with (1.2)), we see that (1.3) becomes (1.1). Therefore, the problem of the existence of standing waves of (1.3) has been reduced to that on the existence of homoclinic solutions of (1.1).

In order to overcome the difficulties caused by the unboundedness of $Z^{m}$ and the lack of periodic conditions, we make some suitable assumptions and get the following result.

Theorem 1.1 The problem (1.1) has infinitely many nontrivial homoclinic solutions if $f_{n}(-s)=-f_{n}(s)$ for all $(n, s) \in Z^{m} \times R$ and the following conditions hold:

$\left(V_{1}\right) \quad V=\left\{v_{n}\right\}_{n \in Z^{m}}$ is bounded from below and satisfies

$$
\lim _{|n| \rightarrow+\infty} v_{n}=+\infty
$$

$\left(F_{1}\right) f_{n} \in C(R, R), f_{n}(s)=o(s)$ as $s \rightarrow 0$, and there exist $a_{1}>0$ and $v>2$ such that

$$
\left|f_{n}(s)\right| \leq a_{1}\left(1+|s|^{\nu-1}\right), \quad \forall(n, s) \in Z^{m} \times R .
$$

(F) $\lim _{|s| \rightarrow+\infty} \frac{F_{n}(s)}{|s|^{2}}=+\infty, \forall n \in Z^{m}$, where $F_{n}(s):=\int_{0}^{s} f_{n}(t) d t,(n, s) \in Z^{m} \times R$.

$\left(F_{3}\right)$ There exist two positive constants $b$ and $\varrho>\max \{1, v-2\}$ such that

$$
\liminf _{|s| \rightarrow+\infty} \frac{f_{n}(s) s-2 F_{n}(s)}{|s|^{\varrho}} \geq b, \quad \forall n \in Z^{m} .
$$

$\left(F_{4}\right) \frac{1}{2} f_{n}(s) s>F_{n}(s)$ if $s \neq 0, F_{n}(s) \geq 0, \forall(n, s) \in Z^{m} \times R$, and

$$
\liminf _{|s| \rightarrow 0} \frac{f_{n}(s) s-2 F_{n}(s)}{|s|^{\imath}} \geq a_{2} \text { for some } a_{2}>0 \text { and } \iota \in[1, v], \forall n \in Z^{m} .
$$

To explain the rationality of the assumptions for the nonlinear terms $f_{n}$, we give the following example. It is easy to check that the functions given in the following example satisfy our assumptions.

Example 1.1 Let

$$
F_{n}(s)=a_{n}\left(|s|^{p}+(p-2)|s|^{p-\varepsilon} \sin ^{2}\left(|s|^{\varepsilon} / \varepsilon\right)\right), \quad s \in R,
$$

where $a_{n} \geq C>0$ for all $n \in Z^{m}, p>2$, and $0<\varepsilon<p-2$. Note that

$$
f_{n}(s) s-2 F_{n}(s)=(p-2) a_{n}\left[(p-2-\varepsilon)|s|^{p-\varepsilon} \sin ^{2}\left(|s|^{\varepsilon} / \varepsilon\right)+\left(1+\sin \left(2|s|^{\varepsilon} / \varepsilon\right)\right)|s|^{p}\right] .
$$


Remark 1.1 Our result extends some results $[15,18,20,21,23]$ in infinite $m$ dimensional lattices.

(1) The results $[15,18,20,21]$ are all about the positive definite case $(\omega<\inf \sigma(-\Delta+V))$, but the temporal frequency $\omega \in R$ in our paper.

(2) The authors of $[15,18,20,21]$ all used the conditions $\left(V_{1}\right),\left(F_{1}\right)$, and $\left(F_{2}\right)$. Besides, the authors of $[15,18]$ also used the following monotony condition:

$$
\frac{f_{n}(s)}{s} \text { is increasing for } s>0 \text { and decreasing for } s<0 .
$$

The authors [20,21] also used the following Ambrosetti-Rabinowitz superlinear condition: there exists $v>2$ such that

$$
0<\nu F_{n}(s) \leq f_{n}(s) s, \quad \forall s \in R \backslash\{0\}
$$

But we use local conditions $\left(F_{3}\right)$ and $\left(F_{4}\right)$ to replace the conditions (1.5) and (1.6). The functions of Example 1.1 satisfy our conditions $\left(F_{1}\right)-\left(F_{4}\right)$, but they do not satisfy (1.5) and (1.6), which shows that our conditions are weaker than the above conditions.

(3) The results in [23] also rely on the monotony condition (1.5).

In Section 2, we establish the variational framework of (1.1) and give some preliminary lemmas. In Section 3, we give the detailed proof of our main result.

\section{Preliminary lemmas}

Let

$$
\begin{aligned}
& l^{p} \equiv l^{p}\left(Z^{m}\right):=\left\{u=\left\{u_{n}\right\}: n \in Z^{m}, u_{n} \in R,\|u\|_{l^{p}}=\left(\sum_{n \in Z^{m}}\left|u_{n}\right|^{p}\right)^{1 / p}<\infty\right\}, \\
& \quad p \in[1,+\infty),
\end{aligned}
$$

be real sequence spaces. Clearly, the following elementary embedding relations hold:

$$
l^{p} \subset l^{q}, \quad\|u\|_{l^{q}} \leq\|u\|_{l^{p}}, \quad 1 \leq p \leq q \leq \infty, \text { where }\|u\|_{l^{\infty}}:=\max _{n \in Z^{m}}\left|u_{n}\right|
$$

Let $L:=-\triangle+V$ be defined by $L u_{n}:=-\triangle u_{n}+v_{n} u_{n}$ for $u \in l^{2}$. Let $E$ be the form domain of $L$, i.e., $E:=\mathcal{D}\left(L^{1 / 2}\right)$ (the domain of $L^{1 / 2}$ ). Under our assumptions, the operator $L$ is an unbounded self-adjoint operator in $l^{2}$. Since the operator $-\triangle$ is bounded in $l^{2}$, it is easy to see that $E=\left\{u \in l^{2}: V^{1 / 2} u \in l^{2}\right\}$, where $V^{1 / 2} u$ is defined by $V^{1 / 2} u_{n}:=v_{n}^{1 / 2} u_{n}$ for $u \in l^{2}$. We define, respectively, on $E$ the inner product and norm by

$$
(u, v)_{E}:=(u, v)_{l^{2}}+\left(L^{1 / 2} u, L^{1 / 2} v\right)_{l^{2}} \quad \text { and } \quad\|u\|_{E}=(u, u)_{E}^{1 / 2} \text {, }
$$

where $(u, v)_{l^{2}}$ is the inner product in $l^{2}$. Then $E$ is a Hilbert space.

Lemma 2.1 ([21]) If (1.4) holds, then we have:

(1) The embedding maps from $E$ into $l^{p}$ are compact, $\forall p \in[2, \infty]$. 
(2) The spectrum $\sigma(L)$ is discrete and consists of simple eigenvalues accumulating to $+\infty$.

By Lemma 2.1(2), we can assume that

$$
\lambda_{1}-\omega<\lambda_{2}-\omega<\cdots<\lambda_{k}-\omega<\cdots \rightarrow+\infty
$$

are all eigenvalues of $L-\omega$ and $e_{k}$ is the associated normalized eigenfunction with the eigenvalue $\lambda_{k}-\omega$ for each $k$, i.e., $(L-\omega) e_{k}=\left(\lambda_{k}-\omega\right) e_{k}$ and $\left\|e_{k}\right\|_{l^{2}}=1, k=1,2, \ldots$ Moreover, $\left\{e_{k}: k=1,2, \ldots\right\}$ is an orthonormal basis of $l^{2}$. Let $\sharp(D)$ denote the number $i$ with $i \in D$. Let

$$
k_{1}:=\sharp\left(\left\{i: \lambda_{i}-\omega<0\right\}\right), \quad k_{0}:=\sharp\left(\left\{i: \lambda_{i}-\omega=0\right\}\right), \quad k_{2}:=k_{0}+k_{1},
$$

and

$$
E^{-}:=\operatorname{span}\left\{e_{1}, \ldots, e_{k_{1}}\right\}, \quad E^{0}:=\operatorname{span}\left\{e_{k_{1}+1}, \ldots, e_{k_{2}}\right\}, \quad E^{+}:=\overline{\operatorname{span}\left\{e_{k_{2}+1}, \ldots\right\}},
$$

where the closure is taken with respect to the norm $\|\cdot\|_{E}$. Then one has the orthogonal decomposition

$$
E=E^{-} \oplus E^{0} \oplus E^{+}
$$

with respect to the inner product $(\cdot, \cdot)_{E}$. Now, we introduce, respectively, on $E$ the following inner product and norm:

$$
(u, v):=\left(u^{0}, v^{0}\right)_{l^{2}}+\left(L^{\frac{1}{2}} u, L^{\frac{1}{2}} v\right)_{l^{2}}, \quad\|u\|=(u, u)^{\frac{1}{2}},
$$

where $u, v \in E=E^{-} \oplus E^{0} \oplus E^{+}$with $u=u^{-}+u^{0}+u^{+}$and $v=v^{-}+v^{0}+v^{+}$. Clearly, the norms $\|\cdot\|$ and $\|\cdot\|_{E}$ are equivalent, and the decomposition $E=E^{-} \oplus E^{0} \oplus E^{+}$is also orthogonal with respect to both inner products $(\cdot, \cdot)$ and $(\cdot, \cdot)_{l^{2}}$.

From the above arguments, we consider the functional $\Phi$ on $E$ defined by

$$
\begin{aligned}
\Phi(u) & =\frac{1}{2}((L-\omega) u, u)_{l^{2}}-\sum_{n \in Z^{m}} F_{n}\left(u_{n}\right) \\
& =\frac{1}{2}\left\|u^{+}\right\|^{2}-\frac{1}{2}\left\|u^{-}\right\|^{2}-I(u),
\end{aligned}
$$

where $I(u):=\sum_{n \in Z^{m}} F_{n}\left(u_{n}\right)$. Under our assumptions, $I, \Phi \in C^{1}(E, R)$, and the derivatives are given by

$$
\left\langle\Phi^{\prime}(u), v\right\rangle=\left(u^{+}, v^{+}\right)-\left(u^{-}, v^{-}\right)-\left\langle I^{\prime}(u), v\right\rangle, \quad\left\langle I^{\prime}(u), v\right\rangle=\sum_{n \in Z^{m}} f_{n}\left(u_{n}\right) v_{n},
$$

where $u, v \in E=E^{-} \oplus E^{0} \oplus E^{+}$with $u=u^{-}+u^{0}+u^{+}$and $v=v^{-}+v^{0}+v^{+}$. The standard argument shows that nonzero critical points of $\Phi$ are nontrivial solutions of (1.1). We shall use the following critical point theorem to prove our main result. 
Lemma 2.2 ([24]) Let $E=\overline{\bigoplus_{j=1}^{\infty} X_{j}} \quad\left(\operatorname{dim} X_{j}<\infty, \forall j \in N\right)$ be a Banach space with the norm $\|\cdot\|, Y_{k}=\bigoplus_{j=1}^{k} X_{j}$, and $Z_{k}=\overline{\bigoplus_{j=k}^{\infty} X_{j}}$. Let the functional $\Phi_{\lambda}=A(u)-\lambda B(u) \in C^{1}: E \rightarrow R$, $\lambda \in[1,2]$. Assume that $\Phi_{\lambda}$ satisfies

(F) $\Phi_{\lambda}$ maps bounded sets to bounded sets for $\lambda \in[1,2]$, and $\Phi_{\lambda}(-u)=\Phi_{\lambda}(u), \forall(\lambda, u) \in$ $[1,2] \times E$.

$\left(F_{2}\right) \quad B(u) \geq 0, \forall u \in E, A(u) \rightarrow \infty$ or $B(u) \rightarrow \infty$ as $\|u\| \rightarrow \infty$.

$\left(F_{3}\right)$ There exist $r_{k}>\rho_{k}>0$ such that

$$
\alpha_{k}(\lambda):=\inf _{u \in Z_{k},\|u\|=\rho_{k}} \Phi_{\lambda}(u)>\beta_{k}(\lambda):=\max _{u \in Y_{k},\|u\|=r_{k}} \Phi_{\lambda}(u), \quad \forall \lambda \in[1,2] .
$$

Then

$$
\alpha_{k}(\lambda) \leq \zeta_{k}(\lambda):=\inf _{\gamma \in \Gamma_{k}} \max _{u \in B_{k}} \Phi_{\lambda}(\gamma(u)), \quad \forall \lambda \in[1,2]
$$

where $B_{k}:=\left\{u \in Y_{k}:\|u\| \leq r_{k}\right\}$ and $\Gamma_{k}:=\left\{\gamma \in C\left(B_{k}, E\right) \mid \gamma\right.$ is odd, $\left.\left.\gamma\right|_{\partial B_{k}}=i d\right\}$. Moreover, for a.e. $\lambda \in[1,2]$, there exists a sequence $\left\{u^{k m}(\lambda)\right\}_{m=1}^{\infty}$ such that

$$
\sup _{m}\left\|u^{k m}(\lambda)\right\|<\infty, \quad \Phi_{\lambda}^{\prime}\left(u^{k m}(\lambda)\right)=0 \quad \text { and } \quad \Phi_{\lambda}\left(u^{k m}(\lambda)\right) \rightarrow \zeta_{k}(\lambda) \quad \text { as } m \rightarrow \infty .
$$

Note that $\operatorname{dim} E^{0}$ and $\operatorname{dim} E^{-}$are finite, we choose an orthonormal basis $\left\{e_{j}\right\}_{j=1}^{k_{1}}$ of $E^{-}$, an orthonormal basis $\left\{e_{j}\right\}_{j=k_{1}+1}^{k_{2}}$ of $E^{0}$, and an orthonormal basis $\left\{e_{j}\right\}_{j=k_{2}+1}^{\infty}$ of $E^{+}$, where $k_{1}$ and $k_{2}$ are defined in (2.1). Then $\left\{e_{j}\right\}_{j=1}^{\infty}$ is an orthonormal basis of $E$. Let $X_{j}:=R e_{j}$, then $Y_{k}=\bigoplus_{m=1}^{k} X_{m}=\operatorname{span}\left\{e_{1}, \ldots, e_{k}\right\}$ and $Z_{k}=\overline{\bigoplus_{m=k}^{\infty} X_{m}}=\overline{\operatorname{span}\left\{e_{k}, \ldots\right\}}$ for all $k \in N$. In order to apply Lemma 2.2 to prove our main result, we define the functionals $A, B$, and $\Phi_{\lambda}$ on $E$ by

$$
A(u)=\frac{1}{2}\left\|u^{+}\right\|^{2}, \quad B(u)=\frac{1}{2}\left\|u^{-}\right\|^{2}+\sum_{n \in Z^{m}} F_{n}\left(u_{n}\right)
$$

and

$$
\Phi_{\lambda}(u)=A(u)-\lambda B(u)=\frac{1}{2}\left\|u^{+}\right\|^{2}-\lambda\left(\frac{1}{2}\left\|u^{-}\right\|^{2}+\sum_{n \in Z^{m}} F_{n}\left(u_{n}\right)\right), \quad \forall u \in E, \forall \lambda \in[1,2] .
$$

Clearly, $\Phi_{\lambda} \in C^{1}(E, R), \forall \lambda \in[1,2]$.

Lemma 2.3 If $\left(F_{4}\right)$ holds, then $\left(F_{2}\right)$ in Lemma 2.2 holds.

Proof Obviously, $B(u) \geq 0$ for all $u \in E$ by $\left(F_{4}\right)$ and the definition of $B(u)$. From the Fact 1 in the Appendix, we see that there is a constant $\epsilon>0$ such that

$$
\sharp\left(\left\{n \in Z^{m}:\left|u_{n}\right| \geq \epsilon\|u\|\right\}\right) \geq 1, \quad \forall u \in H \backslash\{0\},
$$

for any finite-dimensional subspace $H \subset E$. Let $\Lambda_{u}:=\left\{n \in Z^{m}:\left|u_{n}\right| \geq \epsilon\|u\|\right\}, \forall u \in H \backslash\{0\}$. Then by (2.2),

$$
\sharp\left(\Lambda_{u}\right) \geq 1, \quad \forall u \in H \backslash\{0\} .
$$


$\left(F_{2}\right)$ implies that there are $R_{1}, R_{2}>0$ such that

$$
F_{n}(s) \geq R_{1}|s|^{2}, \quad \forall(n, s) \in Z^{m} \times R \text { with }|s| \geq R_{2} .
$$

For any $u \in H$ with $\|u\| \geq R_{2} / \epsilon$, we have

$$
\left|u_{n}\right| \geq R_{2}, \quad \forall n \in \Lambda_{u}
$$

Note that $F_{n}(s) \geq 0$ for all $(n, s) \in Z^{m} \times R$, it follows from (2.3)-(2.5) and the definitions of $B(u)$ and $\Lambda_{u}$ that, for any $u \in H$ with $\|u\| \geq R_{2} / \epsilon$,

$$
\begin{aligned}
B(u) & =\frac{1}{2}\left\|u^{-}\right\|^{2}+\sum_{n \in Z^{m}} F_{n}\left(u_{n}\right) \\
& \geq \sum_{n \in \Lambda_{u}} F_{n}\left(u_{n}\right) \\
& \geq \sum_{n \in \Lambda_{u}} R_{1}\left|u_{n}\right|^{2} \\
& \geq R_{1} \epsilon^{2}\|u\|^{2} \cdot \sharp\left(\Lambda_{u}\right) \geq R_{1} \epsilon^{2}\|u\|^{2} .
\end{aligned}
$$

It implies

$$
B(u) \rightarrow \infty \quad \text { as }\|u\| \rightarrow \infty \text { on } E^{-} \oplus E^{0},
$$

which is due to $E^{-} \oplus E^{0}$ being of finite dimension. It follows from the fact $E=E^{-} \oplus E^{0} \oplus E^{+}$ and the definitions of $A$ and $B$ that we have

$$
A(u) \rightarrow \infty \quad \text { or } \quad B(u) \rightarrow \infty \quad \text { as }\|u\| \rightarrow \infty, \forall u \in E .
$$

The proof is completed.

Lemma 2.4 If the assumptions in Theorem 1.1 are satisfied, then $\left(F_{3}\right)$ in Lemma 2.2 holds.

Proof (a) Note that $\left(F_{1}\right)$ implies that for any $\varepsilon>0$ there exists $C_{\varepsilon}$ such that

$$
\left|F_{n}(s)\right| \leq \varepsilon|s|^{2}+C_{\varepsilon}|s|^{\nu}, \quad \forall(n, s) \in Z^{m} \times R .
$$

It follows from the definition of $\Phi_{\lambda}$ that

$$
\begin{aligned}
\Phi_{\lambda}(u) & \geq \frac{1}{2}\|u\|^{2}-2 \sum_{n \in Z^{m}} F_{n}\left(u_{n}\right) \\
& \geq \frac{1}{2}\|u\|^{2}-2 \sum_{n \in Z^{m}}\left(\varepsilon\left|u_{n}\right|^{2}+C_{\varepsilon}\left|u_{n}\right|^{\nu}\right), \quad \forall(\lambda, u) \in[1,2] \times E^{+} .
\end{aligned}
$$

Let

$$
l_{2}(k):=\sup _{u \in Z_{k} \backslash\{0\}} \frac{\|u\|_{l^{2}}}{\|u\|}, \quad l_{v}(k):=\sup _{u \in Z_{k} \backslash\{0\}} \frac{\|u\|_{l^{\nu}}}{\|u\|}, \quad \forall k \in N .
$$


Note that

$$
l_{2}(k) \rightarrow 0, \quad l_{v}(k) \rightarrow 0 \quad \text { as } k \rightarrow \infty,
$$

which will be proved in the appendix. Obviously, $Z_{k} \subset E^{+}$for all $k \geq k_{2}+1\left(k_{2}+1\right.$ is defined above Lemma 2.3), thus it follows from (2.6)-(2.7) that for any $k \geq k_{2}+1$ we have

$$
\Phi_{\lambda}(u) \geq \frac{1}{2}\|u\|^{2}-2 \varepsilon l_{2}^{2}(k)\|u\|^{2}-2 C_{\varepsilon} l_{\nu}^{\nu}(k)\|u\|^{\nu}, \quad \forall(\lambda, u) \in[1,2] \times Z_{k} .
$$

Let

$$
\rho_{k}:=\left(1-16 \varepsilon l_{2}^{2}(k)\right)\left(16 C_{\varepsilon} l_{v}^{\nu}(k)\right)^{\frac{1}{2-\nu}}
$$

By (2.8), there exists a large enough $k_{3}>k_{2}+1$ such that

$$
0<16 \varepsilon l_{2}^{2}(k)<1, \quad \forall k>k_{3}
$$

By (2.8), (2.10), (2.11), and $v>2$, we have

$$
\rho_{k} \rightarrow \infty \quad \text { as } k \rightarrow \infty
$$

By (2.9)-(2.11), we have

$$
\alpha_{k}(\lambda):=\inf _{u \in Z_{k},\|u\|=\rho_{k}} \Phi_{\lambda}(u) \geq \rho_{k}^{2} / 4>0, \quad \forall k \geq k_{3} .
$$

(b) Note that $Y_{k}$ is of finite dimension, thus (2.2) implies that for any $k \in N$ there exists a constant $\epsilon_{k}>0$ such that

$$
\sharp\left(\left\{n \in Z^{m}:\left|u_{n}\right| \geq \epsilon_{k}\|u\|\right\}\right) \geq 1, \quad \forall u \in Y_{k} \backslash\{0\} .
$$

By $\left(F_{2}\right)$, for any $k \in N$, there exists a constant $S_{k}>0$ such that

$$
F_{n}(s) \geq \frac{|s|^{2}}{\epsilon_{k}^{2}}, \quad \forall(n, s) \in Z^{m} \times R \text { with }|s| \geq S_{k} .
$$

For any $k \in N$ and $u \in Y_{k}$ with $\|u\| \geq S_{k} / \epsilon_{k}$, by (2.13), (2.14), and the fact $F_{n}(s) \geq 0$, we have

$$
\begin{aligned}
\Phi_{\lambda}(u) & \leq \frac{1}{2}\left\|u^{+}\right\|^{2}-\sum_{n \in Z^{m}} F_{n}\left(u_{n}\right) \\
& \leq \frac{1}{2}\|u\|^{2}-\sum_{n \in\left\{n \in Z^{m}:\left|u_{n}\right| \geq \epsilon_{k}\|u\|\right\}} \frac{\left|u_{n}\right|^{2}}{\epsilon_{k}^{2}} \\
& \leq \frac{1}{2}\|u\|^{2}-\frac{\epsilon_{k}^{2}\|u\|^{2}}{\epsilon_{k}^{2}} \cdot \sharp\left(\left\{n \in Z^{m}:\left|u_{n}\right| \geq \epsilon\|u\|\right\}\right) \\
& \leq \frac{1}{2}\|u\|^{2}-\|u\|^{2}=-\frac{1}{2}\|u\|^{2}, \quad \forall \lambda \in[1,2] .
\end{aligned}
$$


Now for any $k \in N$, if we choose

$$
r_{k}>\max \left\{\rho_{k}, S_{k} / \epsilon_{k}\right\}
$$

then (2.15) implies that

$$
\beta_{k}(\lambda):=\max _{u \in Y_{k},\|u\|=r_{k}} \Phi_{\lambda}(u) \leq-r_{k}^{2} / 2<0, \quad \forall k \in N .
$$

Therefore, the proof is finished.

\section{Proof of the main result}

Proof of Theorem 1.1 It is easy to check that $\left(F_{1}\right)$ of Lemma 2.2 holds. Besides, $\left(F_{2}\right)$ and $\left(F_{3}\right)$ hold for all $k \geq k_{3}$ by Lemmas 2.3 and 2.4. Thus Lemma 2.2 implies that for any $k \geq k_{3}$ and a.e. $\lambda \in[1,2]$ there exists a sequence $\left\{u_{i}^{k}(\lambda)\right\}_{i=1}^{\infty} \subset E$ such that

$$
\sup _{i}\left\|u_{i}^{k}(\lambda)\right\|<\infty, \quad \Phi_{\lambda}^{\prime}\left(u_{i}^{k}(\lambda)\right)=0 \quad \text { and } \quad \Phi_{\lambda}\left(u_{i}^{k}(\lambda)\right) \rightarrow \zeta_{k}(\lambda) \quad \text { as } i \rightarrow \infty
$$

where

$$
\zeta_{k}(\lambda):=\inf _{\gamma \in \Gamma_{k}} \max _{u \in B_{k}} \Phi_{\lambda}(\gamma(u)), \quad \forall \lambda \in[1,2]
$$

with $B_{k}:=\left\{u \in Y_{k}:\|u\| \leq r_{k}\right\}$ and $\Gamma_{k}:=\left\{\gamma \in C\left(B_{k}, E\right) \mid \gamma\right.$ is odd, $\left.\left.\gamma\right|_{\partial B_{k}}=i d\right\}$. Furthermore, it follows from the proof of Lemma 2.4 that

$$
\zeta_{k}(\lambda) \in\left[\bar{\alpha}_{k}, \bar{\zeta}_{k}\right], \quad \forall k \geq k_{3}
$$

where $\bar{\zeta}_{k}:=\max _{u \in B_{k}} \Phi_{1}(u)$ and $\bar{\alpha}_{k}:=\rho_{k}^{2} / 4 \rightarrow \infty$ as $k \rightarrow \infty$ by (2.12). By (3.1), for each $k \geq k_{3}$, there exist $\lambda_{j} \rightarrow 1$ as $j \rightarrow \infty$ and $\left\{u_{i}^{k}\left(\lambda_{j}\right)\right\}_{i=1}^{\infty} \subset E$ such that

$$
\begin{aligned}
& \sup _{i}\left\|u_{i}^{k}\left(\lambda_{j}\right)\right\|<\infty, \quad \Phi_{\lambda_{j}}^{\prime}\left(u_{i}^{k}\left(\lambda_{j}\right)\right)=0 \quad \text { and } \quad \Phi_{\lambda_{j}}\left(u_{i}^{k}\left(\lambda_{j}\right)\right) \rightarrow \zeta_{k}\left(\lambda_{j}\right) \\
& \quad \text { as } i \rightarrow \infty
\end{aligned}
$$

Claim $1\left\{u_{i}^{k}\left(\lambda_{j}\right)\right\}_{i=1}^{\infty}$ in (3.3) has a strong convergent subsequence.

Proof Note that $\sup _{i}\left\|u_{i}^{k}\left(\lambda_{j}\right)\right\|<\infty$ for each $k \geq k_{3}$, without loss of generality, we may assume

$$
\begin{aligned}
& \left(u_{i}^{k}\left(\lambda_{j}\right)\right)^{-} \rightarrow\left(u_{j}^{k}\right)^{-}, \quad\left(u_{i}^{k}\left(\lambda_{j}\right)\right)^{0} \rightarrow\left(u_{j}^{k}\right)^{0} \text { and }\left(u_{i}^{k}\left(\lambda_{j}\right)\right)^{+} \rightarrow\left(u_{j}^{k}\right)^{+} \\
& \quad \text { as } i \rightarrow \infty, \forall j \in N,
\end{aligned}
$$

for some $u_{j}^{k}=\left(u_{j}^{k}\right)^{-}+\left(u_{j}^{k}\right)^{0}+\left(u_{j}^{k}\right)^{+} \in E=E^{-}+E^{0}+E^{+}$since $\operatorname{dim}\left(E^{-} \oplus E^{0}\right)<\infty$. By virtue of the Riesz representation theorem, $\Phi_{\lambda_{j}}^{\prime}: E \rightarrow E^{*}$ and $I^{\prime}: E \rightarrow E^{*}$ can be viewed as $\Phi_{\lambda_{j}}^{\prime}$ : 
$E \rightarrow E$ and $I^{\prime}: E \rightarrow E$, respectively, where $E^{*}$ is the dual space of $E$. Note that (3.3) implies that for each $k \geq k_{3}$

$$
0=\Phi_{\lambda_{j}}^{\prime}\left(u_{i}^{k}\left(\lambda_{j}\right)\right)=\left(u_{i}^{k}\left(\lambda_{j}\right)\right)^{+}-\lambda_{j}\left[\left(u_{i}^{k}\left(\lambda_{j}\right)\right)^{-}+I^{\prime}\left(u_{i}^{k}\left(\lambda_{j}\right)\right)\right], \quad \forall i, j \in N
$$

that is,

$$
\left(u_{i}^{k}\left(\lambda_{j}\right)\right)^{+}=\lambda_{j}\left[\left(u_{i}^{k}\left(\lambda_{j}\right)\right)^{-}+I^{\prime}\left(u_{i}^{k}\left(\lambda_{j}\right)\right)\right], \quad \forall i, j \in N
$$

By the standard argument (see $[25,26])$, we know $I^{\prime}: E \rightarrow E^{*}$ is compact. Therefore, $I^{\prime}$ : $E \rightarrow E$ is also compact. It follows from (3.4) and (3.5) that the right-hand side of (3.5) converges strongly in $E$. Combining this with (3.4), we have

$$
\lim _{i \rightarrow \infty} u_{i}^{k}\left(\lambda_{j}\right)=u_{j}^{k} \in E, \quad \forall j \in N \text { and } k \geq k_{3} .
$$

So Claim 1 is true.

By (3.2), (3.3), and (3.6), we have

$$
\Phi_{\lambda_{j}}^{\prime}\left(u_{j}^{k}\right)=0 \quad \text { and } \quad \Phi_{\lambda_{j}}\left(u_{j}^{k}\right) \in\left[\bar{\alpha}_{k}, \bar{\zeta}_{k}\right], \quad \forall j \in N \text { and } k \geq k_{3}
$$

In fact, we can see $\left\{u_{j}^{k}\right\}_{j=1}^{\infty}$ is bounded in $E$, which will be proved in the appendix. Besides, by a similar proof to Claim 1 , we can also see that $\left\{u_{j}^{k}\right\}_{j=1}^{\infty}$ possesses a strong convergent subsequence in $E$ for all $k \geq k_{3}$. Without loss of generality, we may assume

$$
u_{j}^{k} \rightarrow u^{k} \quad \text { as } j \rightarrow \infty, \forall k \geq k_{3} .
$$

For each $k \geq k_{3}$, by (3.7), the limit $u^{k}$ is just a critical point of $\Phi=\Phi_{1}$ with $\Phi\left(u^{k}\right) \in\left[\bar{\alpha}_{k}, \bar{\zeta}_{k}\right]$. Since $\bar{\alpha}_{k} \rightarrow \infty$ as $k \rightarrow \infty$ in (3.2), we get infinitely many nontrivial critical points of $\Phi$. Therefore, we see that problem (1.1) possesses infinitely many nontrivial homoclinic solutions. The proof of Theorem 1.1 is completed.

\section{Appendix}

Fact 1 The result (2.2) holds.

Proof If not, for any $j \in N$, there exists $u^{j} \in H \backslash\{0\}$ such that

$$
\sharp\left(\left\{n \in Z^{m}:\left|u_{n}^{j}\right| \geq\left\|u^{j}\right\| / j\right\}\right)=0 .
$$

Let $v^{j}:=\frac{u^{j}}{\left\|w^{j}\right\|} \in H$, then $\left\|v^{j}\right\|=1$ and

$$
\sharp\left(\left\{n \in Z^{m}:\left|v_{n}^{j}\right| \geq 1 / j\right\}\right)=0, \quad \forall j \in N .
$$

Note that since $H$ is finite dimensional, passing to a subsequence if necessary, we may assume $v^{j} \rightarrow v$ in $E$ for some $v \in H$. Evidently, $\|v\|=1$. Note that any two norms on $H$ are 
equivalent, thus by Lemma 2.1(1), we have

$$
\left\|v^{j}-v\right\|_{l^{2}}^{2}=\sum_{n \in Z^{m}}\left|v_{n}^{j}-v_{n}\right|^{2} \rightarrow 0 \quad \text { as } j \rightarrow \infty
$$

The fact that $\|v\|=1$ implies $\|v\|_{l}=\max _{n \in Z^{m}}\left|v_{n}\right|>0$. By the definition of the norm $\|\cdot\|_{l \infty}$, there exists a constant $\delta_{0}>0$ such that

$$
\sharp\left(\left\{n \in Z^{m}:\left|v_{n}\right| \geq \delta_{0}\right\}\right) \geq 1 .
$$

For any $j \in N$, let

$$
\Lambda_{j}:=\left\{n \in Z^{m}:\left|v_{n}^{j}\right|<1 / j\right\} \quad \text { and } \quad \Lambda_{j}^{c}:=Z^{m} \backslash \Lambda_{j}=\left\{n \in Z^{m}:\left|v_{n}^{j}\right| \geq 1 / j\right\} .
$$

Set $\Lambda_{0}:=\left\{n \in Z^{m}:\left|v_{n}\right| \geq \delta_{0}\right\}$. Then for $j$ large enough, by (A.1) and (A.3), we have

$$
\sharp\left(\Lambda_{j} \cap \Lambda_{0}\right) \geq \sharp\left(\Lambda_{0}\right)-\sharp\left(\Lambda_{j}^{c}\right) \geq 1-0=1 .
$$

It follows from the definitions of $\Lambda_{j}$ and $\Lambda_{0}$ that for $j$ large enough we have

$$
\begin{aligned}
\sum_{n \in Z^{m}}\left|v_{n}^{j}-v_{n}\right|^{2} & \geq \sum_{n \in \Lambda_{j} \cap \Lambda_{0}}\left|v_{n}^{j}-v_{n}\right|^{2} \\
& \geq \sum_{n \in \Lambda_{j} \cap \Lambda_{0}}\left|v_{n}\right|\left(\left|v_{n}\right|-2\left|v_{n}^{j}\right|\right) \\
& \geq \delta_{0}\left(\delta_{0}-2 / j\right) \cdot \sharp\left(\Lambda_{j} \cap \Lambda_{0}\right) \\
& \geq \delta_{0}^{2} / 2>0 .
\end{aligned}
$$

This is in contradiction to (A.2). Therefore, (2.2) holds.

Fact 2 The result (2.8) holds.

Proof It is clear that $0<l_{v}(k+1) \leq l_{v}(k)$, so that $l_{v}(k) \rightarrow l \geq 0$ as $k \rightarrow \infty$. For every $k \geq 0$, there exists $u^{k} \in Z_{k}$ such that $\left\|u^{k}\right\|=1$ and $\left\|u^{k}\right\|_{l^{v}}>l_{v}(k) / 2$. By the definition of $Z_{k}, u^{k} \rightarrow 0$ in $E$, then $u^{k} \rightarrow 0$ in $l^{v}$ dues to Lemma 2.1(1). Therefore, we have $l=0$, that is, $l_{v}(k) \rightarrow 0$. Similarly, $l_{2}(k) \rightarrow 0$. Therefore, (2.8) holds.

Fact $3\left\{u_{j}^{k}\right\}_{j=1}^{\infty}$ is bounded in $E$.

Proof Note that $\left(F_{3}\right)$ implies that there exists a constant $L_{0}>0$ such that

$$
\frac{1}{2} f_{n}(s) s-F_{n}(s) \geq \frac{b}{4}|s|^{\varrho}, \quad \forall(n, s) \in Z^{m} \times R \text { with }|s| \geq L_{0} .
$$

For notational simplicity, we will set

$$
u_{j}:=u_{j}^{k}, \quad \forall j \in N \text { and } k \geq k_{3}
$$


throughout this paragraph. Note that $\left(F_{4}\right)$ implies $\frac{1}{2} f_{n}(s) s-F_{n}(s) \geq 0$ for all $(n, s) \in Z^{m} \times R$, it follows from (3.7), (A.4), and the definition of $\Phi_{\lambda}$ that

$$
\begin{aligned}
\Phi_{\lambda_{j}}\left(u_{j}\right) & =\Phi_{\lambda_{j}}\left(u_{j}\right)-\frac{1}{2}\left\langle\Phi_{\lambda_{j}}^{\prime}\left(u_{j}\right), u_{j}\right\rangle \\
& =\lambda_{j} \sum_{n \in Z^{m}}\left[\frac{1}{2} f_{n}\left(u_{j, n}\right) u_{j, n}-F_{n}\left(u_{j, n}\right)\right] \\
& \geq \sum_{n \in \Pi_{j}}\left[\frac{1}{2} f_{n}\left(u_{j, n}\right) u_{j, n}-F_{n}\left(u_{j, n}\right)\right] \\
& \geq \frac{b}{4} \sum_{n \in \Pi_{j}}\left|u_{j, n}\right|^{\varrho}, \quad \forall j \in N,
\end{aligned}
$$

where $\Pi_{j}:=\left\{n \in Z^{m}:\left|u_{j, n}\right| \geq L_{0}\right\}$. It follows from (3.7) that

$$
\sum_{n \in \Pi_{j}}\left|u_{j, n}\right|^{\varrho} \leq D_{1}, \quad \forall j \in N
$$

for some $D_{1}>0$. Note that $\left(F_{4}\right)$ implies that there exists a constant $L_{1} \in\left(0, L_{0}\right)$ such that

$$
\frac{1}{2} f_{n}(s) s-F_{n}(s) \geq \frac{a_{2}}{4}|s|^{\ell}, \quad \forall(n, s) \in Z^{m} \times R \text { with }|s| \leq L_{1} .
$$

Similar to (A.5), by (A.7), $\left(F_{1}\right)$, and the fact $\frac{1}{2} f_{n}(s) s-F_{n}(s)>0$ if $s \neq 0$ (see $\left(F_{4}\right)$ ), we get

$$
\begin{aligned}
\Phi_{\lambda_{j}}\left(u_{j}\right)= & \lambda_{j} \sum_{n \in Z^{m}}\left[\frac{1}{2} f_{n}\left(u_{j, n}\right) u_{j, n}-F_{n}\left(u_{j, n}\right)\right] \\
\geq & \sum_{n \in Z^{m} \backslash \Pi_{j}}\left[\frac{1}{2} f_{n}\left(u_{j, n}\right) u_{j, n}-F_{n}\left(u_{j, n}\right)\right] \\
= & \sum_{\left\{n \in Z^{m}:\left|u_{j, n}\right| \leq L_{1}\right\}}\left[\frac{1}{2} f_{n}\left(u_{j, n}\right) u_{j, n}-F_{n}\left(u_{j, n}\right)\right] \\
& +\sum_{\left\{n \in Z^{m}: L_{1} \leq\left|u_{j, n}\right|<L_{0}\right\}}\left[\frac{1}{2} f_{n}\left(u_{j, n}\right) u_{j, n}-F_{n}\left(u_{j, n}\right)\right] \\
\geq & \sum_{\left\{n \in Z^{m}:\left|u_{j, n}\right| \leq L_{1}\right\}} \frac{a_{2}}{4}\left|u_{j, n}\right|^{l}+\sum_{\left\{n \in Z^{m}: L_{1} \leq\left|u_{j, n}\right|<L_{0}\right\}} D_{2}\left|u_{j, n}\right|^{l} \\
\geq & \sum_{\left\{n \in Z^{m}:\left|u_{j, n}\right|<L_{0}\right\}} D_{3}\left|u_{j, n}\right|^{l}=\sum_{n \in Z^{m} \backslash \Pi_{j}} D_{3}\left|u_{j, n}\right|^{l}, \quad \forall j \in N,
\end{aligned}
$$

for some $D_{2}, D_{3}>0$. It follows from (3.7) that

$$
\sum_{n \in Z^{m} \backslash \Pi_{j}}\left|u_{j, n}\right|^{l} \leq D_{4}, \quad \forall j \in N
$$

for some $D_{4}>0$. For any $j \in N$, let $\chi_{j}: Z^{m} \rightarrow R$ be the indicator of $\Pi_{j}$, that is,

$$
\chi_{j, n}:= \begin{cases}1, & d n \in \Pi_{j}, \quad \forall j \in N . \\ 0, & n \notin \Pi_{j},\end{cases}
$$


Then by (A.6) and the definitions of $\Pi_{j}$ and $\chi_{j}$, we have

$$
\left\|\left(1-\chi_{j}\right) u_{j}\right\|_{l^{\infty}} \leq L_{0} \quad \text { and } \quad\left\|\chi_{j} u_{j}\right\|_{l^{\varrho}}^{\varrho}=\sum_{n \in \Pi_{j}}\left|u_{j, n}\right|^{\varrho} \leq D_{1}, \quad \forall j \in N
$$

It follows from the equivalence of any two norms on the finite-dimensional space $E^{0} \oplus E^{-}$ and Hölder's inequality that

$$
\begin{aligned}
\left\|u_{j}^{-}+u_{j}^{0}\right\|_{l^{2}}^{2} & =\left(u_{j}^{-}+u_{j}^{0}, u_{j}\right)_{l^{2}} \\
& =\left(u_{j}^{-}+u_{j}^{0},\left(1-\chi_{j}\right) u_{j}\right)_{l^{2}}+\left(u_{j}^{-}+u_{j}^{0}, \chi_{j} u_{j}\right)_{l^{2}} \\
& \leq\left\|\left(1-\chi_{j}\right) u_{j}\right\|_{l^{\infty}} \cdot\left\|u_{j}^{-}+u_{j}^{0}\right\|_{l^{1}}+\left\|\chi_{j} u_{j}\right\|_{l} \cdot\left\|u_{j}^{-}+u_{j}^{0}\right\|_{\ell^{\prime}} \\
& \leq L_{0} c_{1}\left\|u_{j}^{-}+u_{j}^{0}\right\|_{l^{2}}+D_{1}^{1 / \varrho} c_{2}\left\|u_{j}^{-}+u_{j}^{0}\right\|_{l^{2}} \\
& =\left(L_{0} c_{1}+D_{1}^{1 / \varrho} c_{2}\right)\left\|u_{j}^{-}+u_{j}^{0}\right\|_{l^{2}}, \quad \forall j \in N,
\end{aligned}
$$

for some $c_{1}, c_{2}>0$, where $\varrho^{\prime}:=\frac{\varrho}{\varrho-1}$. Consequently, we get

$$
\left\|u_{j}^{-}+u_{j}^{0}\right\|_{l^{2}} \leq L_{0} c_{1}+D_{1}^{1 / \varrho} c_{2}, \quad \forall j \in N
$$

By the equivalence of norms $\|\cdot\|_{l^{2}}$ and $\|\cdot\|$ on $E^{0} \oplus E^{-}$, we know there exists $c_{3}>0$ such that

$$
\left\|u_{j}^{-}+u_{j}^{0}\right\| \leq c_{3}, \quad \forall j \in N
$$

Therefore,

$$
\left\|u_{j}^{-}\right\| \leq c_{3}, \quad \forall j \in N
$$

By the definition of $\Phi_{\lambda_{j}}$, we have

$$
\left\|u_{j}^{+}\right\|^{2}=2 \Phi_{\lambda_{j}}\left(u_{j}\right)+\lambda_{j}\left\|u_{j}^{-}\right\|^{2}+2 \lambda_{j} \sum_{n \in Z^{m}} F_{n}\left(u_{j, n}\right), \quad \forall j \in N .
$$

Note that $\left(F_{1}\right)$ implies that for any $\varepsilon>0$ there exists $C_{\varepsilon}$ such that

$$
\left|F_{n}(s)\right| \leq \varepsilon|s|^{2}+C_{\varepsilon}|s|^{\nu}, \quad \forall(n, s) \in Z^{m} \times R .
$$

Therefore, by (3.7), (A.8)-(A.12), and the Sobolev embedding theorem we have

$$
\begin{aligned}
\left\|u_{j}\right\|^{2} & =\left\|u_{j}^{-}+u_{j}^{0}\right\|^{2}+\left\|u_{j}^{+}\right\|^{2} \\
& =\left\|u_{j}^{-}+u_{j}^{0}\right\|^{2}+2 \Phi_{\lambda_{j}}\left(u_{j}\right)+\lambda_{j}\left\|u_{j}^{-}\right\|^{2}+2 \lambda_{j} \sum_{n \in Z^{m}} F_{n}\left(u_{j, n}\right) \\
& \leq c_{4}+2 \lambda_{j} \sum_{n \in Z^{m}}\left(\varepsilon\left|u_{j, n}\right|^{2}+C_{\varepsilon}\left|u_{j, n}\right|^{v}\right) \\
& =c_{4}+2 \lambda_{j} \varepsilon\left\|u_{j}\right\|_{l^{2}}^{2}+2 \lambda_{j} C_{\varepsilon}\left(\sum_{n \in Z^{m} \backslash \Pi_{j}}\left|u_{j, n}\right|^{v}+\sum_{n \in \Pi_{j}}\left|u_{j, n}\right|^{v}\right)
\end{aligned}
$$




$$
\begin{aligned}
& \leq c_{4}+c_{5} \varepsilon\left\|u_{j}\right\|^{2}+c_{6} L_{0}^{\nu-\iota} \sum_{n \in Z^{m} \backslash \Pi_{j}}\left|u_{j, n}\right|^{\iota}+c_{6} \sum_{n \in \Pi_{j}}\left|u_{j, n}\right|^{v} \\
& \leq c_{4}+c_{5} \varepsilon\left\|u_{j}\right\|^{2}+c_{6} L_{0}^{\nu-\iota} D_{4}+c_{6} \sum_{n \in \Pi_{j}}\left|u_{j, n}\right|^{\nu}, \quad \forall j \in N,
\end{aligned}
$$

for some $c_{4}, c_{5}, c_{6}>0$, where $\iota \leq v$ is defined in $\left(F_{4}\right)$. If $v-\varrho \geq 0$, by (A.6) and Lemma 2.1(1), we have

$$
\sum_{n \in \Pi_{j}}\left|u_{j, n}\right|^{\nu} \leq\left\|u_{j}\right\|_{l^{\infty}}^{\nu-\varrho} \sum_{n \in \Pi_{j}}\left|u_{j, n}\right|^{\varrho} \leq D_{1} c_{7}\left\|u_{j}\right\|^{\nu-\varrho}, \quad \forall j \in N,
$$

for some $c_{7}>0$. If $v-\varrho<0$, by (A.6) and the definition of $\Pi_{j}$, we have

$$
\sum_{n \in \Pi_{j}}\left|u_{j, n}\right|^{v}=\sum_{n \in \Pi_{j}} \frac{\left|u_{j, n}\right|^{\varrho}}{\left|u_{j, n}\right|^{\varrho-v}} \leq \frac{1}{L_{0}^{\varrho-v}} \sum_{n \in \Pi_{j}}\left|u_{j, n}\right|^{\varrho} \leq \frac{D_{1}}{L_{0}^{\varrho-v}}, \quad \forall j \in N
$$

Note that $v-\varrho<2\left(\right.$ see $\left.\left(F_{3}\right)\right)$ and $\varepsilon>0$ is arbitrary, thus it follows from (A.13)-(A.15) that $\left\{u_{j}\right\}$ is bounded in $E$.

\section{Competing interests}

The authors declare that they have no competing interests.

\section{Authors' contributions}

All authors contributed equally to the writing of this paper. All authors read and approved the final manuscript.

\section{Acknowledgements}

Research supported by National Natural Science Foundation of China (No. 11401011).

We would like to thank the editors and referees for their valuable comments which have led to an improvement of the presentation of this paper.

Received: 19 September 2016 Accepted: 17 October 2016 Published online: 26 October 2016

\section{References}

1. Kopidakis, G, Aubry, S, Tsironis, GP: Targeted energy transfer through discrete breathers in nonlinear systems. Phys. Rev. Lett. 87, 165501 (2001)

2. Christodoulides, DN, Lederer, F, Silberberg, Y: Discretizing light behaviour in linear and nonlinear waveguide lattices. Nature 424, 817-823 (2003)

3. Livi, R, Franzosi, R, Oppo, GL: Self-localization of Bose-Einstein condensates in optical lattices via boundary dissipation. Phys. Rev. Lett. 97, 060401 (2006)

4. Chen, G, Ma, S: Discrete nonlinear Schrödinger equations with superlinear nonlinearities. Appl. Math. Comput. 218 5496-5507 (2012)

5. Chen, G, Ma, S: Ground state and geometrically distinct solitons of discrete nonlinear Schrödinger equations with saturable nonlinearities. Stud. Appl. Math. 131, 389-413 (2013)

6. Chen, G, Ma, S: Homoclinic solutions of discrete nonlinear Schrödinger equations with asymptotically or super linear terms. Appl. Math. Comput. 232, 787-798 (2014)

7. Pankov, A: Gap solitons in periodic discrete nonlinear equations. Nonlinearity 19, 27-40 (2006)

8. Pankov, A: Gap solitons in periodic discrete nonlinear Schrödinger equations. II. A generalized Nehari manifold approach. Discrete Contin. Dyn. Syst. 19, 419-430 (2007)

9. Pankov, A, Rothos, V: Periodic and decaying solutions in discrete nonlinear Schrödinger with saturable nonlinearity. Proc. R. Soc. A 464, 3219-3236 (2008)

10. Pankov, A: Gap solitons in periodic discrete nonlinear Schrödinger equations with saturable nonlinearities. J. Math. Anal. Appl. 371, 254-265 (2010)

11. Yang, M, Chen, W, Ding, Y: Solutions for discrete periodic Schrödinger equations with spectrum 0. Acta Appl. Math. 110, 1475-1488 (2010)

12. Zhou, Z, Yu, J, Chen, Y: On the existence of gap solitons in a periodic discrete nonlinear Schrödinger equation with saturable nonlinearity. Nonlinearity 23, 1727-1740 (2010)

13. Shi, H, Zhang, H: Existence of gap solitons in periodic discrete nonlinear Schrödinger equations. J. Math. Anal. Appl. 361, 411-419 (2010)

14. Shi, H: Gap solitons in periodic discrete Schrödinger equations with nonlinearity. Acta Appl. Math. 109, 1065-1075 (2010)

15. Ma, D, Zhou, Z: Existence and multiplicity results of homoclinic solutions for the DNLS equations with unbounded potentials. Abstr. Appl. Anal. 2012, 703596 (2012) 
16. Pankov, A, Zhang, G: Standing wave solutions for discrete nonlinear Schrödinger equations with unbounded potentials and saturable nonlinearity. J. Math. Sci. 177, 71-82 (2011)

17. Pankov, A: Standing waves for discrete nonlinear Schrödinger equations: sign-changing nonlinearities. Appl. Anal. 92 , 308-317 (2013)

18. Sun, G: On standing wave solutions for discrete nonlinear Schrödinger equations. Abstr. Appl. Anal. 2013, 436919 (2013)

19. Zhang, G, Pankov, A: Standing waves of the discrete nonlinear Schrödinger equations with growing potentials. Commun. Math. Anal. 5, 38-49 (2008)

20. Zhang, G, Liu, F: Existence of breather solutions of the DNLS equations with unbounded potentials. Nonlinear Anal. 71, 786-792 (2009)

21. Zhang, G: Breather solutions of the discrete nonlinear Schrödinger equations with unbounded potentials. J. Math Phys. 50, 013505 (2009)

22. Zhang, G, Pankov, A: Standing wave solutions of the discrete non-linear Schrödinger equations with unbounded potentials, II. Appl. Anal. 89, 1541-1557 (2010)

23. Zhou, Z, Ma, D: Multiplicity results of breathers for the discrete nonlinear Schrödinger equations with unbounded potentials. Sci. China Math. 58, 781-790 (2015)

24. Zou, W: Variant fountain theorems and their applications. Manuscr. Math. 104, 343-358 (2001)

25. Benci, V, Rabinowitz, PH: Critical point theorems for indefinite functionals. Invent. Math. 52, 241-273 (1979)

26. Rabinowitz, PH: Minimax Methods in Critical Point Theory with Applications to Differential Equations. CBMS Reg. Conf. Ser. Math., vol. 65. Am. Math. Soc. Providence (1986)

\section{Submit your manuscript to a SpringerOpen ${ }^{\ominus}$ journal and benefit from:}

- Convenient online submission

- Rigorous peer review

- Immediate publication on acceptance

- Open access: articles freely available online

- High visibility within the field

Retaining the copyright to your article 